\begin{tabular}{llll} 
Abstract S21 Table 1 & Characteristics & & \\
\hline & COPD & HV & HS \\
\hline Age (yrs) [Mean \pm SD] & $58 \pm 1$ & $50 \pm 11$ & $55 \pm 1$ \\
Sex (\% Female) & 22 & 46 & 63 \\
Richness [Mean \pm SD] & $71 \pm 16$ & $69 \pm 13$ & $69 \pm 16$ \\
SW [Mean \pm SD] & $1.90 \pm 0.63$ & $2.66 \pm 0.51$ & $2.51 \pm 0.34$ \\
FEV $_{1}$ [Mean \pm SD] & $1.46 \pm 0.45$ & $3.12 \pm 0.55$ & $3.20 \pm 0.71$ \\
FEV $_{1} \%$ Predicted [Mean \pm SD] & $58 \pm 17$ & $98 \pm 6$ & $95 \pm 13$ \\
FEV $_{1} /$ FVC Ratio [Mean \pm SD] & $0.51 \pm 0.08$ & $0.82 \pm 0.06$ & $0.79 \pm 0.06$ \\
\hline
\end{tabular}

in the lower airways of patients with COPD, healthy non-smokers and smokers.

Methods Bronchial wash samples were collected from patients with COPD (GOLD 1-3; $\mathrm{n}=18$ ), healthy non-smokers (HV; $\mathrm{n}$ $=11$ ) and healthy smokers (HS; $\mathrm{n}=8$ ). Samples were processed using the Illumina MiSeq platform. The Shannon-Wiener Index (SW) of diversity, lung obstruction $\left(\mathrm{FEV}_{1} / \mathrm{FVC}\right.$ ratio) and ordination by Non-Metric Multidimensional Scaling (NMDS) on Bray-Curtis dissimilarity indices were analysed to evaluate how samples were related. Principal component analysis (PCA) was performed to assess the effect specific taxa had within each cohort. Characteristics of each cohort are shown in Table 1.

Results There was no difference in taxa richness between cohorts (range: 69-71; $p=0.954$ ). Diversity (SW Index) was significantly lower in COPD samples compared to samples from HV and HS ( $p=0.009$ and $p=0.033$, respectively). There was no significant difference between HV and HS ( $p=0.186)$. The $\mathrm{FEV}_{1} / \mathrm{FVC}$ ratio was significantly lower for COPD compared to HV $\left(p=9 * 10^{-8}\right)$ and HS $\left(p=2 * 10^{-6}\right)$, respectively. NMDS analysis showed that communities belonging to either of the healthy groups were more similar to each other than they were to samples belonging to the COPD group. PCA analysis showed that members of Streptococcus sp. and Haemophilus sp. had the largest effect on the variance explained in COPD. In HS, Haemophilus sp., Fusobaterium sp., Actinomyces sp., Prevotella sp. and Veillonella sp. had the largest effect on the variance explained, while in HV Neisseria sp., Porphyromonas sp., Actinomyces sp., Atopobium sp., Prevotella and Veillonella sp. had the largest effect on the variance explained.

Conclusions The study demonstrates that microbial communities in the lower airways of patients with COPD are significantly different from that seen in healthy comparison groups. Patients with COPD had lower microbial diversity than either of the healthy comparison groups, higher relative abundance of members of Streptococcus sp. and lower relative abundance of a number of key anaerobes.

\section{S22 THE EFFECT OF CIGARETTE SMOKE ON IMPORTANT PATHOGENS IN COPD LUNG INFECTION}

K McGown, MM Tunney, SJ McGrath, JS Elborn, DF Gilpin. Queens University Belfast, Belfast, UK

\subsection{6/thoraxjnl-2014-206260.28}

Introduction The leading cause of COPD in developed nations is exposure to tobacco smoke. COPD is characterised by acute periods of exacerbation, which are often bacterial in aetiology. The direct effect of cigarette smoke on bacteria present in the COPD lung, and how this may drive disease progression, has not been determined. This preliminary study aimed to determine the effect of cigarette smoke extract (CSE) on the growth and antibiotic susceptibility of COPD bacterial lung pathogens.
Methods CSE was prepared as described previously. Briefly, smoke from one, two, three or four cigarettes was bubbled through $100 \mathrm{mls}$ growth medium. Bacterial type strains (Pseudomonas aeruginosa, Moraxella catarrhalis, Streptococcus pneumoniae, Prevotella spp and Haemophilus influenzae) were inoculated into growth medium +/- CSE and incubated either aerobically or anaerobically (Prevotella spp). Total viable counts (TVC cfu/ml) were estimated from 0-48 hrs (aerobes) and 0-72 hrs (Prevotella spp). Changes in minimum inhibitory concentration (MIC) of antibiotics used in the treatment of respiratory infections were determined by E-Test ${ }^{\circledR}$, in bacterial cultures exposed daily to CSE over 12 days.

Results The growth of P.aeruginosa, S. pneumoniae and H. influenzae were not completely inhibited by any concentration of CSE; however a reduction in growth rate at higher concentrations was observed. M. catarrhalis growth was completely inhibited by two cigarettes $/ 100 \mathrm{ml}$ growth medium. No difference in growth was observed between Prevotella spp +/- CSE. A marked increase in P.aeruginosa resistance to tetracycline and doxycycline was observed after repeated CSE exposure: resistance to tetracycline and doxycycline increased from 24 to $>256 \mu \mathrm{g} / \mathrm{ml}$, and 48 $>256 \mu \mathrm{g} / \mathrm{ml}$, respectively.

Conclusions The growth of principal bacteria isolated from COPD patients were not affected by concentrations of CSE utilised in this study, but changes in the susceptibility of $P$. aeruginosa to tetracyclines was observed. This increase in resistance may be mediated by efflux pump up-regulation, and may lead to crossresistance with other antibiotics. Work currently underway aims to determine whether CSE induces other key phenotypic changes (virulence factor expression and/or biofilm production) which might enhance the pathogenicity of these bacteria in the presence of CSE and result in poorer outcomes for patients with COPD.

\section{Sleep disordered breathing - assessment and treatment}

\section{S23 RESULTS OF A NATIONAL SURVEY OF PRE-OPERATIVE SCREENING FOR OBSTRUCTIVE SLEEP APNOEA}

${ }^{1} \mathrm{R}$ Sharrock, ${ }^{2} \mathrm{~S}$ Baudouin, ${ }^{1} \mathrm{~S}$ West. ${ }^{1}$ Freeman Hospital, Newcastle Upon Tyne, UK; ${ }^{2}$ Royal Victoria Infirmary, Newcastle Upon Tyne, UK

\subsection{6/thoraxjnl-2014-206260.29}

Introduction and objectives There is currently no UK guidance (from BTS, BSS or RCA) regarding screening for obstructive sleep apnoea in the pre-operative setting. Evidence suggests that undiagnosed OSA is associated with increased post-operative complications but no trials have examined whether screening the UK's general surgical population is justifiable. We sought to examine current UK practice and opinion in this regard.

Methods A postal survey was sent to all 180 UK sleep service providers asking whether they had a hospital policy for pre-operative screening for OSA and what this consisted of. If there was no policy they were asked how pre-operative patients with suspected OSA were identified. Further details regarding diagnostic confirmation and opinion regarding practice were sought.

Results We received 84 replies. There is a spectrum of current practice amongst respondents. There were 31 centres (37\%) with a policy for screening for OSA. Of these, $42 \%$ screened all patients with a questionnaire e.g. STOP BANG, 23\% screened only patients undergoing certain operations, $13 \%$ screened patients with high BMI only. Of those with a policy who 
estimated the number of referred patients, $60 \%$ saw more than $>5$ per month. Of centres with no policy only $26 \%$ estimated that they received $>5$ referrals per month. Without a policy $72 \%$ of referrals came from clinical suspicion alone.

Overall $96 \%$ of respondents felt that all patients at high risk of OSA should be screened for OSA. 36 respondents thought it would be ethical to randomise identified cases of OSA to a potential trial of peri-operative CPAP or no CPAP, compared with 40 who did not.

Conclusions There is no established UK standard practice for screening for OSA pre-operatively, despite a majority opinion amongst questionnaire responders that high risk patients should be. There would be cost implications if National pre-operative OSA screening was implemented and there therefore needs to be clear evidence based benefit before proceeding.

\section{S24 REPEATABILITY AND EFFECT OF INCENTIVES ON AN OFFICE BASED ADVANCED DRIVING SIMULATOR (MINIUOLDS) TO ASSESS DRIVING PERFORMANCE IN OBSTRUCTIVE SLEEP APNOEA SYNDROME (OSAS)}

${ }^{1} \mathrm{~A}$ Dwarakanath, ${ }^{2} \mathrm{SL}$ Jamson, ${ }^{3} \mathrm{PD}$ Baxter, 'MW Elliott. 'St. James' University Hospital, Leeds, UK; ${ }^{2}$ Institute for Transport Studies, University of Leeds, Leeds, UK; ${ }^{3}$ Division of Biostatistics, LIGHT, Centre for Epidemiology and Biostatistics, University of Leeds, Leeds, UK

\subsection{6/thoraxjnl-2014-206260.30}

Introduction Some patients with OSAS are at higher risk of being involved in road traffic accidents. No objective tests have been shown to predict reliably whether an individual is safe to drive or not and there is significant variation in the advice given by the clinicians. Using continuously measured variables in an advanced PC-based driving simulator the at risk patients can be identified with a high degree of accuracy.

We have investigated whether this finding is repeatable. Individuals may "raise their game" if they know that their licence is at stake. We have therefore also investigated the effect of an incentive on the test.

Methods 150 untreated OSAS patients (males-131) were randomised to either the repeatability $(\mathrm{n}=50)$ or incentive arm $(\mathrm{n}=$ 100). All performed a simulator run, after initial acclimatisation. In the repeatability arm, patients performed the simulator run on two separate occasions with no knowledge of the results. In the incentive arm, patients performed the simulator run on two separate occasions but just prior to the second run were told about their performance and offered a prize if they could improve their performance by $10 \%$.

SDLP in epoch 3 and "veer" reaction time (Veer-RT) were the co-primary outcome variables. Classification of patients into "pass", "fail" and "indeterminate" were the secondary outcome variables. Results were analysed using paired and unpaired $\mathrm{T}$ tests with the level of significance set at $\mathrm{p}<0.05$.

Results 137 patients (repeatability arm-48, incentive arm-89) completed the trial. The median duration between the two simulator runs was 13 days (range, 5-55). SDLP in epoch 3 and Veer-RT were repeatable (P- 0.54, $\Delta$ SDLP- 0.01 and P- $0.37, \Delta$ Veer-RT- 0.13) respectively. There was no effect of an incentive on SDLP in epoch 3 (P-0.18) and Veer-RT (P-0.57). There was no difference in the simulator outcome between the two runs [pass (P- 0.70), indeterminate (0.06), fail (P- 0.16)].

Conclusions SDLP and Veer-RT are consistent between runs on the MiniUoLDS and this is not affected by a simple incentive. Advanced office PC based simulators may be helpful when advising patients with OSAS about driving.
S25 SLEEPY SNORERS WITH "FLOW LIMITATION SYNDROME": A MISSED OPPORTUNITY FOR CPAP?

${ }^{1} \mathrm{R}$ Yadavilli, 'B Chakrabarti, 'S McDougall, 'L Horne, 'S Emegbo, 'S Craig, ${ }^{1} \mathrm{~N}$ Duffy, ${ }^{1} \mathrm{R}$ Parker, ${ }^{1} \mathrm{~J}$ O'Reilly. 'Aintree Chest Centre, University Hospital Aintree, Liverpool, UK; 'University of Liverpool, Liverpool, UK

\subsection{6/thoraxjnl-2014-206260.31}

Background The apnoea-hypopnoea index (AHI) is used to define Obstructive Sleep Apnoea Syndrome (OSAS). Some subjects however, present primarily with excessive daytime sleepiness (EDS) and loud snoring, but investigation may reveal an elevated Respiratory Disturbance Index (RDI) with most events comprising Flow limitations. Little UK based data exists regarding treatment outcomes in this group.

Methodology/results 118 subjects (mean age 52 years; Epworth sleepiness scale score (ESS) 13.58 (5.30); 80\% male) presented between November 2011-October 2013 to the Sleep Service with EDS as a primary symptom, loud snoring, RDI $>15$ with AHI $\leq 11$ (Mean RDI 21.77 (9.43)); AHI 8.03(2.74); ODI 6.72 (4.49) and were treated with CPAP. At 30 day compliance review, 60\% (71/118) had benefited from CPAP with mean ESS pre-CPAP 14.13 (5.12) falling to 7.70 (4.82) following CPAP. The mean BMI was found to be significantly higher in those 71 subjects benefiting from CPAP (33.20 (SD 8.13) v 30.26 (SD 7.40); p = 0.04) but no significant differences were noted in baseline Epworth score, age, gender, AHI, RDI, ODI and Pulse Transit Time (PTT).

This "Flow Limitation" cohort was compared with 261 subjects (mean age 56 years; ESS 12.47(5.61); 82\%Male) diagnosed with OSAS during the same time period (Mean AHI 37.11 (19.94); mean ODI 31.15 (19.74) and treated with CPAP. 76\% $(199 / 261)$ of the OSAS group reported benefit from CPAP; ESS fell from 13.24 (5.35) to 6.60 (4.74) following CPAP therapy.

Comparing the "Flow Limitation" group with the "OSA" group, the mean BMI (32.03(7.94) v 34.70(8.65); p = 0.004) and age $(51.75(12.34)$ v 56.20(12.18); $\mathrm{p}=0.001)$ were significantly lower in the "Flow Limitation" subjects but no significant difference was noted in baseline ESS. Those deriving benefit from CPAP in the OSA group demonstrated significantly higher CPAP usage $(4.45(2.24)$ v $3.83(2.15)$ hours/night; $\mathrm{p}=0.04)$.

Conclusion Basing treatment decisions on AHI rather than RDI may miss a proportion of patients exhibiting similar levels of EDS as those with OSAS who would otherwise have gained benefit from CPAP. Despite the observed benefit, CPAP usage appeared lower in this "Flow Limitation" cohort who appeared overall to be a younger group with a lower BMI compared to those with OSA.

\section{S26 WHAT ARE THE PREDICTORS OF DEVELOPING HYPOVENTILATION IN OBESITY?}

${ }^{1}$ A Manuel, ${ }^{2} \mathrm{~N}$ Hart, ${ }^{1} \mathrm{~J}$ Stradling. 'Oxford Centre for Respiratory Medicine, Oxford Biomedical Research Centre, Churchill Campus, Oxford University Hospitals NHS Trust, Oxford, OX3 7LJ, UK, Oxford, UK; '² Lane Fox Clinical Respiratory Physiology Centre, St Thomas' Hospital, London, UK

\subsection{6/thoraxjnl-2014-206260.32}

Introduction Obesity Hypoventilation Syndrome (OHS) is conventionally defined by the combination of obesity (BMI $>30 \mathrm{~kg}$ / $\left.\mathrm{m}^{2}\right)$ and daytime hypercapnia $\left(\mathrm{PaCO}_{2}>6 \mathrm{kPa}\right.$, with no alternative explanation); sleep-disordered breathing may or may not be included in the definition. The development of ventilatory failure in obese individuals is highly variable, and the additional factors responsible have not been comprehensively studied. In obese 